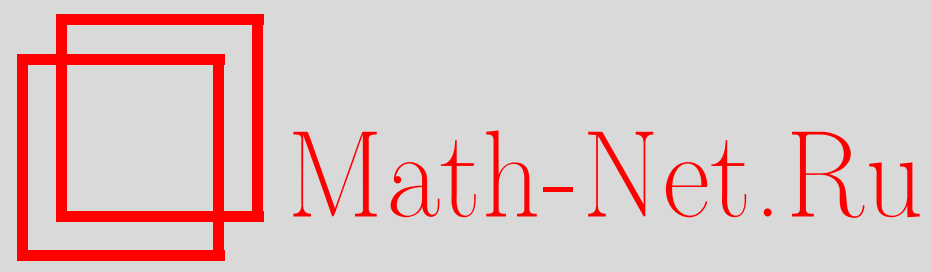

М. Ю. Планида, Асимптотики собственных элементов оператора Лапласа со сменой типа граничного условия на узкой уплощенной полосе, Матем. заметки, 2004, том 75, выпуск 2, 236-252

DOI: https://doi.org/10.4213/mzm30

Использование Общероссийского математического портала Math-Net.Ru подразумевает, что вы прочитали и согласны с пользовательским соглашением http://www.mathnet.ru/rus/agreement

Параметры загрузки:

IP : 3.89 .197 .203

26 апреля 2023 г., 05:17:02

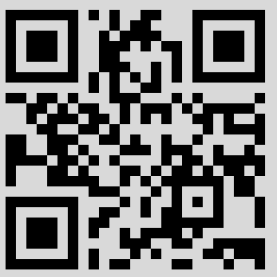




\title{
АСИМПТОТИКИ СОБСТВЕННЫХ ЭЛЕМЕНТОВ ОПЕРАТОРА ЛАПЛАСА СО СМЕНОЙ ТИПА ГРАНИЧНОГО УСЛОВИЯ НА УЗКОЙ УПЛОЩЕННОЙ ПОЛОСЕ
}

М. Ю. Планида

\begin{abstract}
В работе методом согласования асимптотических разложений строятся асимптотики по малому параметру собственных значений и собственных функций оператора Лапласа в области со сменой типа граничного условия на узкой уплощенной полосе при условии, что на узкой полосе границы задается условие Неймана, а на остальной части границы - условие Дирихле. Мальм параметром является ширина полосы.

Библиографоя: 15 названий.
\end{abstract}

1. Постановка задачи. Построению асимптотик решений эллиптических краевых задач в областях с вырезанными малыми отверстиями и узкими щелями посвящено значительное число работ (см., например, [1] и содержащийся в этой монографии обзор литературы). Асимптотики собственных значений краевых задач для оператора Лапласа в таких областях были получены в [2]-[7]. В [8]-[10] были построены асимптотики собственных значений и собственных функций задачи Дирихле со сменой типа граничного условия на участке границы, стягивающемся к точке. В [11] рассматривалась задача Синьорини со сменой типа граничного условия на узкой полосе в случае, когда в окрестности этой полоски граница совпадает с цилиндрической поверхностью, а сама полоска стягивается к кривой, образующейся при сечении этой цилиндрической поверхности перпендикулярной плоскостью. В настоящей работе смена типа граничного условия осуществляется на узкой уплощенной полоске, стягивающейся к замкнутой кривой. Для такой краевой задачи ниже строятся асимптотики собственных значений и собственных функций по малому параметру, характеризующему ширину узкой полоски.

Пусть $x=\left(x_{1}, x_{2}, x_{3}\right), \Omega \subset \mathbb{R}^{3}$ - односвязная ограниченная область с бесконечно дифференцируемой границей $\Gamma, \gamma \in C^{\infty}$ - замкнутая кривая без самопересечений на плоскости $x_{3}=0, \gamma \subset \Gamma$ и $\Omega$ в окрестности кривой $\gamma$ лежит в полупространстве $x_{3}>0$. В окрестности $\gamma$ введем координаты $\left(y_{1}, y_{2}, s\right)$, где $s$ - натуральньй параметр на кривой $\gamma, y_{2}=x_{3}, y_{1}-$ расстояние до $\gamma$ на плоскости $x_{3}=0$, измеренное вдоль внешней нормали к кривой $\gamma$ (понимаемой как граница двумерной области в плоскости $x_{3}=0$ ). Положим

$$
\gamma_{\varepsilon}=\left\{\left(y_{1}, 0, s\right): \varepsilon f_{1}(s)<y_{1}<\varepsilon f_{2}(s)\right\}, \quad \Gamma_{\varepsilon}=\Gamma \backslash \bar{\gamma}_{\varepsilon},
$$

Работа выполнена при поддержке Российского фонда фундаментальных исследований, проект № 02-01-00693, программы "Ведушие научные школы" и программы "Университеты России", проект № УР.04.01.010. 


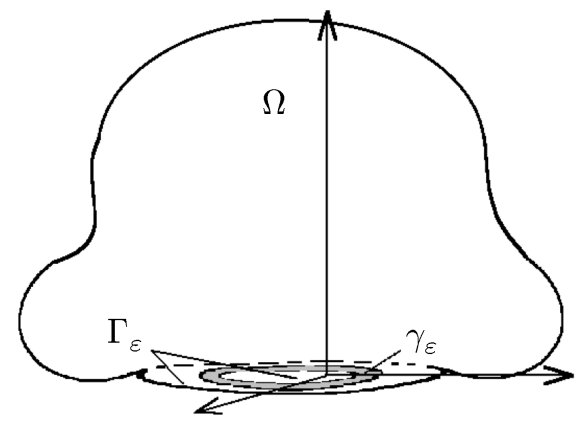

Рис. 1

где $f_{1}(s), f_{2}(s) \in C^{\infty}(\gamma), 0<\varepsilon \ll 1$ (см. рис. 1$)$.

В [12] доказано следующее утверждение

Лемма 1.1. Пусть $\lambda_{0}$ - простое собственное значение краевой задачи

$$
-\Delta \psi_{0}=\lambda_{0} \psi_{0} \quad \text { в } \Omega, \quad \psi_{0}=0 \quad \text { на } \Gamma .
$$

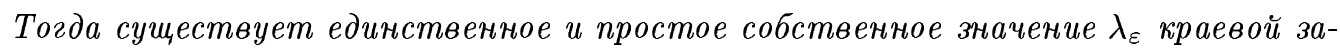
дачи

$$
-\Delta \psi_{\varepsilon}=\lambda_{\varepsilon} \psi_{\varepsilon} \quad{ }_{\boldsymbol{\theta}} \Omega, \quad \psi_{\varepsilon}=0 \quad \text { на } \Gamma_{\varepsilon}, \quad \frac{\partial \psi_{\varepsilon}}{\partial \tau}=0 \quad \text { на } \gamma_{\varepsilon},
$$

әде $\tau$ - внешняя нормаль, сходящееся при $\varepsilon \rightarrow 0 \kappa \lambda_{0}$, а для нормированных в $L_{2}(\Omega)$ собственных функиий имеет место сильная сходимость $\psi_{\varepsilon} \rightarrow \psi_{0}$ в $L_{2}(\Omega)$ и слабая сходимость в $W_{2}^{1}(\Omega)$.

В работе методом согласования асимптотических разложений [1] строятся асимптотики при $\varepsilon \rightarrow 0$ собственного значения краевой задачи $(1.2)$, сходящегося к простому собственному значению краевой задачи (1.1), и соответствующей собственной функции. Всюду далее $\lambda_{0}$ - простое собственное значение краевой задачи (1.1).

Структура работы выглядит следующим образом. В п. 2 исследуются решения задачи Дирихле с заданньми особенностями на кривой $\gamma$, необходимые при построении асимптотик собственных элементов краевой задачи (1.2). Асимптотики этих элементов строятся методом согласования асимптотических разложений в п. 3 . Пункт 4 посвящен обоснованию построенньх асимптотик. В заключительном п. 5 приводятся примеры и даются некоторые приложения построенных асимптотик.

2. Решения задачи Дирихле с особенностями на кривой $\gamma$. Нетрудно проверить, что при достаточно малых $y=\left(y_{1}, y_{2}\right)$ оператор Лапласа имеет вид

$$
\Delta=\frac{\partial^{2}}{\partial y_{1}^{2}}+\frac{1}{H} \frac{\partial H}{\partial y_{1}} \frac{\partial}{\partial y_{1}}+\frac{1}{H} \frac{\partial}{\partial s}\left(\frac{1}{H} \frac{\partial}{\partial s}\right)+\frac{\partial^{2}}{\partial y_{2}^{2}}
$$

где $H=-1-y_{1} t(s)$, a $t(s) \in C^{\infty}(\gamma)$. Обозначим через $\Delta_{y}$ двумерный оператор Лапласа в переменных $y$. Тогда

$$
\Delta=\Delta_{y}-\sum_{i=0}^{\infty} b_{i}(s) y_{1}^{i} \frac{\partial}{\partial y_{1}}-\sum_{i=1}^{\infty} h_{i-1}(s) y_{1}^{i-1} \frac{\partial^{2}}{\partial s^{2}}-\sum_{i=0}^{\infty} p_{i+1}(s) y_{1}^{i+1} \frac{\partial}{\partial s}
$$


где $b_{i}, h_{q}, p_{m}-$ некоторые функции из $C^{\infty}(\gamma)$.

Пусть $(r, \varphi)$ - полярные координаты, соответствующие декартовьг координатам $\left(y_{1}, y_{2}\right)$. Обозначим через $Y_{i}(y), X_{i}(y)$ однородные гармонические полиномы вида

$$
Y_{i}(y)=r^{i} \sin (i \varphi), \quad X_{i}(y)=r^{i} \cos (i \varphi),
$$

где $i \geqslant 0$ целые, а $P_{j}^{(q)}(y ; s), Q_{j}^{(q)}(y ; s), P_{j, 1}^{(q)}(y ; s)$ - однородные полиномы степени $j$ по переменной $y$ с коэффициентами, зависящими от $s$.

Лемма 2.1. Пусть функиия $d(s) \in C^{\infty}(\gamma), \mu$ - произвольное действительное число, $k>0$ - иелое число. Тогда существует ряд

$$
\begin{aligned}
& G^{(k)}(y ; s)=\sum_{i=0}^{\infty} g_{i}(y ; s), \\
& g_{0}(y ; s)=d(s) r^{-k} \sin (k \varphi), \\
& g_{1}(y ; s)=\frac{1}{4} d(s) b_{0}(s) r^{-2 k} Y_{k+1} \text {, } \\
& g_{2}(y ; s)=\frac{1}{2}\left(\frac{d(s) b_{0}^{2}(s)}{4}-\frac{d(s) b_{1}(s)}{2}+h_{0}(s) d^{\prime \prime}(s)-\mu d(s)\right) Y_{1} \ln r \\
& -\frac{d(s)}{8}\left(\frac{b_{0}^{2}(s)}{4}-\frac{b_{1}(s)}{2}\right) r^{-2} Y_{3} \quad \text { npu } \quad k=1, \\
& g_{n}(y ; s)=\sum_{j=0}^{n-1} A_{n, j}^{(k)}(s) Y_{k+n-2 j} r^{-2 k+2 j}, \quad 2 \leqslant n \leqslant k, \quad n p u \quad k \neq 1, \\
& g_{k+i}(y ; s)=\sum_{j=0}^{k-1} A_{k+i, j}^{(k)}(s) Y_{2 k+i-2 j} r^{-2 k+2 j}+\ln r \sum_{j=0}^{[i / 2]} B_{k+i, j}^{(k)}(s) Y_{i-2 j} r^{2 j} \\
& +\sum_{j=1}^{[i / 2]} C_{k+i, j}^{(k)}(s) Y_{i-2 j} r^{2 j}, \quad i \geqslant 1
\end{aligned}
$$

əде $A_{m, q}^{(l)}, B_{m, q}^{(l)}, C_{m, q}^{(l)} \in C^{\infty}(\gamma), a b_{q}(s), h_{q}(s)$ - коэффичиенты в $(2.1)$, который $n р и$ $r \rightarrow 0$ является решением уравнения

$$
(\Delta+\mu) g=0 .
$$

ДокАЗАТЕЛЬСТво. При $r \rightarrow 0$ решение уравнения (2.5) будем искать в виде (2.2). Подставляя (2.2) в (2.5), учитьвая (2.1) и вьписьвая отдельно равенства при одинаковых степенях $r$, получаем рекуррентную систему уравнений

$$
\begin{aligned}
\Delta_{y} g_{0}= & 0 \\
\Delta_{y} g_{n}= & \sum_{i=0}^{n-1} b_{i}(s) y_{1}^{i} \frac{\partial g_{n-i-1}}{\partial y_{1}}+\sum_{i=1}^{n-1} h_{i-1}(s) y_{1}^{i-1} \frac{\partial^{2} g_{n-i-1}}{\partial s^{2}} \\
& +\sum_{i=0}^{n-3} p_{i+1}(s) y_{1}^{i+1} \frac{\partial g_{n-i-3}}{\partial s}-\mu g_{n-2}, \quad n \geqslant 1 .
\end{aligned}
$$


Непосредственными вычислениями легко проверить следующие равенства:

$$
\begin{gathered}
\Delta_{y}\left(Z_{j}(y) \ln r\right)=2 r^{-2} j Z_{j}(y), \quad \Delta_{y}\left(r^{\alpha} Z_{j}(y)\right)=\alpha(\alpha+2 j) r^{\alpha-2} Z_{j}(y), \\
\Delta_{y}\left(r^{\alpha} Z_{j}(y) \ln r\right)=r^{\alpha-2} Z_{j}(y)(\alpha(\alpha+2 j) \ln r+2(j+\alpha)), \\
\frac{\partial \ln r}{\partial y_{1}}=r^{-2} X_{1}, \quad \frac{\partial\left(r^{i} Y_{j}\right)}{\partial y_{1}}=\frac{i}{2}\left(r^{i} Y_{j-1}+r^{i-2} Y_{j+1}\right)+j r^{i} Y_{j-1}, \\
y_{1}^{2 m}=\sum_{j=0}^{m} l_{j}^{(2 m)} r^{2(m-j)} X_{2 j}(y), \\
y_{1}^{2 m-1}=\sum_{j=1}^{m} l_{j}^{(2 m-1)} r^{2(m-j)} X_{2 j-1}(y), \\
X_{n} Y_{j}=\frac{1}{2}\left(Y_{n+j}+r^{2 n} Y_{j-n}\right), \quad j \geqslant n, \\
X_{n} Y_{j}=\frac{1}{2}\left(Y_{n+j}-r^{2 j} Y_{n-j}\right), \quad n>j,
\end{gathered}
$$

где $Z_{k}(y)$ - однородный гармонический полином степени $k$, a $l_{i}^{(q)}$ - некоторые постоянные. Первое из уравнений (2.6) выполнено согласно выбору функции $g_{0}(y ; s)$. Формулы $(2.3),(2.4)$ выводятся непосредственно из соотношений $(2.6),(2.7)$. Формулы для остальных $g_{i}(y ; s)$ доказываются индукцией с использованием равенств $(2.6),(2.7)$.

ЗАМЕчАнИЕ 2.1. Из явного вида формул для $g_{i}$ следует, что $g_{i}\left(y_{1}, 0 ; s\right)=0$.

Пусть $\psi_{0}$-нормированная в $L_{2}(\Omega)$ собственная функция краевой задачи $(1.1)$. В силу (2.1) и граничного условия в (1.1) имеет место представление

$$
\psi_{0}(x(y ; s))=\sum_{i=1}^{\infty} P_{i}^{(0)}(y ; s), \quad y \rightarrow 0
$$

где

$$
\begin{gathered}
P_{1}^{(0)}(y ; s)=\alpha_{1}(s) r \sin \varphi, \quad P_{2}^{(0)}(y ; s)=\alpha_{2}(s) r^{2} \sin 2 \varphi \\
P_{3}^{(0)}(y ; s)=\alpha_{3}(s) r^{3} \sin \varphi+D_{1}^{(0)}(s) r^{3} \sin 3 \varphi \\
\alpha_{1}(s)=\frac{\partial \psi_{0}(x(s))}{\partial \tau}, \quad \alpha_{3}(s)=\frac{1}{8}\left(2 \alpha_{2} b_{0}(s)+h_{0}(s) \alpha_{1}^{\prime \prime}(s)-\lambda_{0} \alpha_{1}(s)\right),
\end{gathered}
$$

$\alpha_{2}(s), D_{1}^{(0)}(s)$ - некоторые функции, зависяшие от $\gamma$ и $\Omega$.

Лемма 2.2. Пусть функиия $d(s) \in C^{\infty}(\gamma)$. Существуют постоянная $\Lambda$, определяемая равенством

$$
\Lambda=-\pi \int_{\gamma} \alpha_{1}(s) d(s) d s
$$

и функиия $\Psi(x ; d(s)) \in C^{\infty}(\bar{\Omega} \backslash \gamma)$, являющаяся решением краевой задачи

$$
-\Delta \Psi=\lambda_{0} \Psi+\Lambda \psi_{0}, \quad x \in \Omega, \quad \Psi=0, \quad x \in \Gamma \backslash \gamma,
$$


и имеющая при $r \rightarrow 0$ дифференцируемую асимптотику

$$
\begin{aligned}
\Psi(x ; d(s))= & d(s) r^{-1} \sin \varphi+r^{-2} P_{2}^{(2)}(y ; d(s))+r^{-2} P_{3}^{(2)}(y ; d(s)) \\
& \quad+P_{1,1}^{(2)}(y ; d(s)) \ln r+O\left(r^{2} \ln r\right), \\
P_{2}^{(2)}(y ; d(s))= & \frac{1}{4} d(s) b_{0}(s) Y_{2}, \\
P_{3}^{(2)}(y ; d(s))= & -\frac{1}{8}\left(\frac{d(s) b_{0}^{2}(s)}{4}+\frac{d(s) b_{1}(s)}{2}\right) Y_{3}+D_{1}^{(1)}(s) r^{2} Y_{1}, \\
P_{1,1}^{(2)}(y ; d(s))= & \frac{1}{2}\left(\frac{d(s) b_{0}^{2}(s)}{4}-\frac{d(s) b_{1}(s)}{2}+h_{0}(s) d^{\prime \prime}(s)-\lambda_{0} d(s)\right) Y_{1},
\end{aligned}
$$

где $D_{1}^{(1)}(s) \in C^{\infty}(\gamma)-$ функиия, зависящая от $\gamma u \Omega$.

ДокАЗАТЕЛЬСтво. Пусть $\chi(t)$ - бесконечно дифференцируемая срезающая функция, тождественно равная единице при $t<1$ и нулю при $t>2$, а $\sigma$ - достаточно малое фиксированное положительное число. Будем искать функцию $\Psi(x ; d(s))$ в виде

$$
\Psi(x ; d(s))=g_{N}(x)=\chi\left(r \sigma^{-1}\right) \widehat{G}_{N}^{(1)}(x)+\tilde{g}_{N}(x),
$$

где $\widehat{G}_{N}^{(1)}(x)-N$-я частичная сумма ряда $(2.2)$ при $k=1$, удовлетворяющая лемме 2.1 с $\mu=\lambda_{0}$, а $\tilde{g}_{N}(x)$ - некоторая достаточно гладкая функция. Заметим, что в силу леммы 2.1 (см. замечание 2.1) функция $\chi\left(r \sigma^{-1}\right) \widehat{G}_{N}^{(1)}(x)$ обращается в нуль на $\Gamma \backslash \gamma$. Тогда для $\tilde{g}_{N}(x)$ получаем краевую задачу

$$
\begin{gathered}
-\Delta \tilde{g}_{N}=\lambda_{0} \tilde{g}_{N}+\Lambda(N) \psi_{0}+F_{N}^{1}, \quad x \in \Omega, \quad \tilde{g}_{N}=0, \quad x \in \Gamma, \\
F_{N}^{1}=\Delta \chi \widehat{G}_{N}^{(1)}+2 \sum_{i=1}^{3} \frac{\partial \chi}{\partial x_{i}} \frac{\partial \widehat{G}_{N}^{(1)}}{\partial x_{i}}+\chi\left(\Delta+\lambda_{0}\right) \widehat{G}_{N}^{(1)} .
\end{gathered}
$$

В силу (2.6)

$$
\Delta \widehat{G}_{N}^{(1)}=\sum_{j=0}^{N} y_{1}^{N-j} \phi_{j}^{(1)} \frac{\partial g_{j}}{\partial y_{1}}+\sum_{i=0}^{1} \sum_{j=0}^{N-i-1} y_{1}^{N-j-1} \phi_{j}^{(i+2)} \frac{\partial^{2-i} g_{j}}{\partial s^{2-i}}
$$

где $\phi_{q}^{(m)}(s, y) \in C^{\infty}(\gamma)$. Первые два слагаемых в сумме $(2.16)$ принадлежат классу $C^{\infty}(\bar{\Omega})$, так как $\Delta \widehat{G}_{N}^{(1)} \in C^{\infty}(\bar{\Omega} \backslash \gamma)$, а носители функций $\Delta\left(\chi\left(r \sigma^{-1}\right)\right), \partial \chi\left(r \sigma^{-1}\right) / \partial x_{i}$ отделены от $\gamma$. Далее, в силу выражений для $g_{i}(y ; s)$ из леммы 2.1 и (2.17) вытекает, что

$$
\left(\Delta+\lambda_{0}\right) \widehat{G}_{N}^{(1)}=O\left(r^{N-2} \ln r\right) \quad \text { при } r \rightarrow 0 .
$$

Следовательно, $F_{N} \in C^{N-3}(\bar{\Omega})$. Так как $\lambda_{0}$ - собственное значение предельной задачи, то равенство $\left(F_{N}^{1}+\Lambda(N) \psi_{0}, \psi_{0}\right)_{\Omega}=0$ является необходимым и достаточньм условием разрешимости $(2.15)$, т.е.

$$
\Lambda(N)=-\int_{\Omega} F_{N}^{1} \psi_{0} d x
$$


При таком выборе $\Lambda(N)$ краевая задача (2.15) разрешима, причем $\tilde{g}_{N} \in C^{N-1}(\bar{\Omega})$. Раскладьвая функцию $\tilde{g}_{N}$ по формуле Тейлора и учитывая, что $\tilde{g}_{N}=0$ на $\Gamma$, получаем

$$
\tilde{g}_{N}(x)=\sum_{j=1}^{N-2} Q_{j}^{(1)}(y ; s)+O\left(r^{N-1}\right)
$$

$Q_{1}^{(1)}(y ; s)=D_{1}^{(1)}(s) Y_{1}(y), D_{1}^{(1)}(s) \in C^{N-2}(\gamma)$ - некоторые функции, зависящие от $\gamma$ и $\Omega$. Заметим, что решение (2.15) определено с точностью до собственной функции $\psi_{0}$. Поэтому для определенности положим

$$
\left(g_{N}, \psi_{0}\right)_{\Omega}=0
$$

Покажем, что функция $g_{N}(x)$ не зависит от $N$. Пусть $N_{1} \geqslant N \geqslant 4$. Тогда очевидно, что $\tilde{g}_{N_{1}}(x)-\tilde{g}_{N}(x) \in C^{3}(\bar{\Omega})$. Функция $\chi\left(r \sigma^{-1}\right)\left(\widehat{G}_{N_{1}}^{(1)}(x)-\widehat{G}_{N}^{(1)}(x)\right)$ принадлежит классу $C^{N}(\bar{\Omega})$ в силу $(2.2)$ и леммы 2.1. Таким образом, мы получили, что разность $g_{N, N_{1}}=g_{N_{1}}(x)-g_{N}(x) \in C^{3}(\bar{\Omega})$. Так как каждая из функций $g_{N_{1}}$ и $g_{N}$ удовлетворяет (2.11), то $g_{N, N_{1}}$ является решением этой краевой задачи с $\Lambda=\Lambda\left(N_{1}\right)-\Lambda(N)$ и, значит, $g_{N, N_{1}}$ является собственной функцией. Но в силу нормировки (2.19) получаем, что $g_{N_{1}}=g_{N}$. То есть $g_{N}$ не зависит от $N$. Из $(2.14)$ и $(2.18)$ в силу произволав выборе $N$ получаем (2.12).

Осталось показать справедливость (2.10). Пусть

$$
S_{\mu}^{2}=\left\{x=\left(y_{1}, y_{2}, s\right): s \in \gamma, r<\mu\right\} .
$$

Умножим обе части равенства в $(2.11)$ на $\psi_{0}$, возьмем интеграл по $\Omega \backslash S_{\mu}^{2}$ от обеих частей. Интегрируя полученное равенство по частям, вьводим

$$
\Lambda=\int_{\Omega \cap \partial S_{\mu}^{2}} \psi_{0} \frac{\partial \Psi}{\partial r} d S-\int_{\Omega \cap \partial S_{\mu}^{2}} \frac{\partial \psi_{0}}{\partial r} \Psi d S .
$$

Отсюда, учитьвая $(2.8),(2.9),(2.12)$ и переходя к пределу при $\mu \rightarrow 0$, получаем $(2.10)$. Лемма доказана.

Аналогично доказательству леммы 2.2 , но с заменой ряда $G^{(1)}$ на $G^{(k)}$, доказьвается

Лемма 2.3. Пусть функиия $d(s) \in C^{\infty}(\gamma), k>0-$ иелое число. Тогда существует гармоническая в $\Omega$ функиия $\Psi^{(k)}(x ; d(s))$, обращающаяся в нуль на $Г \curlyvee и$ имеющая при $r \rightarrow 0$ асимптотическое разложение

$$
\Psi^{(k)}(x ; d(s))=G^{(k)}(y ; s)+\sum_{j=1}^{\infty} Q_{j}^{(k)}(y ; s)
$$

әде $Q_{1}^{(k)}(y ; s)=D_{1}^{(k)}(s) Y_{1}(y), \quad D_{1}^{(k)}(s) \in C^{\infty}(\gamma)-$ функиии, зависящие от $\gamma$ и $\Omega, a$ ряд $G^{(k)}(y ; s)$ удовлетворяет утвержсдению леммы 2.1 с $\mu=0$. 
3. Построение формальных асимптотик методом согласования асимптотических разложений. Так как $\psi_{0}$, очевидно, не удовлетворяет граничному условию Неймана на $\gamma_{\varepsilon}$, то в окрестности $\gamma_{\varepsilon}$ введем внутреннее разложение, зависящее от $\varepsilon$ и переменных $\xi=\left(\xi_{1}, \xi_{2}\right)$, где $\xi_{i}=y_{i} \varepsilon^{-1}$. Пусть $\rho=|\xi|$. Переписывая $(2.8)$ в переменных $\xi$, получаем

$$
\psi_{0}(x)=\sum_{j=1}^{3} \varepsilon^{j} P_{j}^{(0)}(\xi ; s)+O\left(\varepsilon^{4} \rho^{4}\right), \quad \varepsilon \rho \rightarrow 0 .
$$

В [12] доказано, что $\psi_{\varepsilon} \rightarrow \psi_{0}$ в $L_{2}(\Omega)$ при $\varepsilon \rightarrow 0$, поэтому, следуя методу согласования асимптотических разложений [1] и учитывая (3.1), приближенное значение собственной функции $\psi_{\varepsilon}$ в малой окрестности кривой $\gamma$ будем искать в виде

$$
\begin{gathered}
\psi_{\varepsilon}(x) \approx \varepsilon v_{1}(\xi ; s), \\
v_{1}(\xi ; s)=P_{1}^{(0)}(\xi ; s)(1+o(1)), \quad \rho \rightarrow \infty .
\end{gathered}
$$

Подставляя $(2.1),(3.2)$ в (1.2), переходя в полученном равенстве к переменньп $\xi$ и вьписьвая коэффициенты при минимальной степени $\varepsilon\left(\mathrm{a}\right.$, именно, при $\left.\varepsilon^{-1}\right)$, получаем следующую краевую задачу:

$$
\Delta_{\xi} v_{1}=0, \quad \xi_{2}>0, \quad v_{1}=0, \quad \xi \in \Gamma_{1}, \quad \frac{\partial v_{1}}{\partial \xi_{2}}=0, \quad \xi \in \gamma_{1},
$$

где

$$
\begin{aligned}
\Gamma_{1} & =\left\{\xi: \xi_{1}<f_{1}(s), \xi_{2}=0\right\} \cup\left\{\xi: \xi_{1}>f_{2}(s), \xi_{2}=0\right\} \\
\gamma_{1} & =\left\{\xi: f_{1}(s)<\xi_{1}<f_{2}(s), \xi_{2}=0\right\} .
\end{aligned}
$$

Пусть $\zeta=\xi_{1}+i \xi_{2}$, где $i-$ мнимая единица. Легко видеть, что функция

$$
v_{1}(\xi ; s)=\alpha_{1}(s) \operatorname{Im} \sqrt{\left(\zeta-f_{1}(s)\right)\left(\zeta-f_{2}(s)\right)}
$$

удовлетворяет (3.3) и имеет на бесконечности асимптотику

$$
v_{1}(\xi ; s)=P_{1}^{(0)}(\xi ; s)+\sum_{j=1}^{5} \beta_{j}(s) \rho^{-j} \sin (j \varphi)+O\left(\rho^{-6}\right), \quad \rho \rightarrow \infty,
$$

где $\beta_{j}(s) \in C^{\infty}(\gamma)$ - некоторые явно выгисляемые функции и, в частности,

$$
\begin{aligned}
& \beta_{1}(s)=\frac{\alpha_{1}(s)}{8}\left(f_{2}(s)-f_{1}(s)\right)^{2}, \quad \beta_{2}(s)=\frac{\beta_{1}(s)}{2}\left(f_{2}(s)+f_{1}(s)\right), \\
& \beta_{3}(s)=\frac{\beta_{1}(s)}{16}\left(5 f_{1}^{2}(s)+5 f_{2}^{2}(s)+6 f_{1}(s) f_{2}(s)\right) .
\end{aligned}
$$

Переписывая асимптотику $\varepsilon v_{1}$ (см. (3.5)) при $\rho \rightarrow \infty$ в переменных $x$ получаем, что при $r \varepsilon^{-1} \rightarrow \infty$

$$
\psi_{\varepsilon}(x) \approx \varepsilon v_{1}(\xi ; s)=P_{1}^{(0)}(y ; s)+\sum_{j=1}^{5} \varepsilon^{j+1} \beta_{j}(s) r^{-j} \sin (j \varphi)+O\left(\varepsilon^{7} r^{-6}\right) .
$$


Поэтому, следуя методу согласования асимптотических разложений, будем искать собственную функцию вне малой окрестности кривой $\gamma$ в виде

$$
\begin{gathered}
\psi_{\varepsilon}(x) \approx \widehat{\psi}_{\varepsilon, 6}(x)=\psi_{0}(x)+\sum_{i=2}^{6} \varepsilon^{i} \psi_{i}(x), \\
\psi_{j+1}(x)=\beta_{j}(s) r^{-j} \sin (j \varphi)(1+o(1)), \quad r \rightarrow 0,
\end{gathered}
$$

а собственное значение $\lambda_{\varepsilon}$ в виде

$$
\lambda_{\varepsilon} \approx \hat{\lambda}_{\varepsilon}=\lambda_{0}+\varepsilon^{2} \lambda_{2}
$$

Подставляя (3.8) и (3.7) в (1.2), приравнивая коэффициенты при степенях $\varepsilon^{m}, m \leqslant 2$, и переходя к пределу в описании $\Gamma_{\varepsilon}$, получаем краевую задачу (1.1) и краевую задачу

$$
-\Delta \psi_{2}=\lambda_{0} \psi_{2}+\lambda_{2} \psi_{0}, \quad x \in \Omega, \quad \psi_{2}=0, \quad x \in \Gamma \backslash \gamma
$$

Из леммы $2.2,(2.9)$ и $(3.6)$ следует, что $\psi_{2}(x)=\Psi\left(x ; \beta_{1}(s)\right)$ является решением краевой задачи (3.9) при

$$
\lambda_{2}=-\frac{\pi}{8} \int_{\gamma}\left(\frac{\partial \psi_{0}}{\partial \tau}\right)^{2}\left(f_{2}-f_{1}\right)^{2} d s
$$

и имеет асимптотику

$$
\begin{aligned}
\psi_{2}(x)= & \beta_{1} r^{-1} \sin \varphi+r^{-2} P_{2}^{(2)}\left(y ; \beta_{1}(s)\right)+r^{-2} P_{3}^{(2)}\left(y ; \beta_{1}(s)\right) \\
& +P_{1,1}^{(2)}\left(y ; \beta_{1}(s)\right) \ln r+O\left(r^{2} \ln r\right)
\end{aligned}
$$

где полиномы $P_{j}^{(2)}, P_{1,1}^{(2)}$ определяются в (2.13).

Положим

$$
\begin{aligned}
\psi_{3}(x) & =\Psi^{(2)}\left(x ; \beta_{2}(s)\right)+\Psi^{(1)}\left(x ; \delta^{1}(s)\right), \\
\psi_{4}(x) & =\Psi^{(3)}\left(x ; \beta_{3}(s)\right)+\Psi^{(2)}\left(x ; \delta^{2}(s)\right), \\
\psi_{q} & =\Psi^{(q-1)}\left(x ; \beta_{q-1}(s)\right) \quad \text { при } q=5,6,
\end{aligned}
$$

где $\delta^{1}(s), \delta^{2}(s)$ - произвольные функции, которые будут определены ниже. Из (3.12), $(2.20)$ и леммы 2.1 следует

$$
\begin{aligned}
& \psi_{3}(x)=\beta_{2}(s) r^{-2} \sin 2 \varphi+r^{-4} P_{3}^{(3)}(y ; s)+\delta^{1}(s) r^{-1} \sin \varphi+O(1), \\
& \psi_{4}(x)=\beta_{3}(s) r^{-3} \sin 3 \varphi+r^{-6} P_{4}^{(4)}(y ; s)+\delta^{2}(s) r^{-2} \sin 2 \varphi+O\left(r^{-1}\right), \\
& \psi_{5}(x)=\beta_{4}(s) r^{-4} \sin 4 \varphi+O\left(r^{-3}\right), \\
& \psi_{6}(x)=\beta_{5}(s) r^{-5} \sin 5 \varphi+O\left(r^{-4}\right), \\
& P_{3}^{(3)}(y ; s)=\frac{1}{4} \beta_{2}(s) b_{0}(s) Y_{3}, \quad P_{4}^{(4)}(y ; s)=\frac{1}{4} \beta_{3}(s) b_{0}(s) Y_{4} .
\end{aligned}
$$


Подставляя (3.1), (3.11) и (3.13) в (3.7), переходя в главных членах асимптотик полученного равенства к переменным $\xi$ и группируя коэффициенты при одинаковых степеня $\varepsilon$ и $\ln \varepsilon$, вьводим

$$
\begin{aligned}
\psi_{\varepsilon}(x) \approx \widehat{\psi}_{\varepsilon, 6}(x)=\varepsilon V_{1}(\xi ; s)+\varepsilon^{2}\left(V_{2}(\xi ; s)+\sum_{j=1}^{2} \delta^{j}(s) \rho^{-j} \sin (j \varphi)\right) \\
+\varepsilon^{3} V_{3}(\xi ; s)+\varepsilon^{3} \ln \varepsilon V_{3,1}(\xi ; s) \\
+O\left(r^{4}+\varepsilon^{2} r^{2}|\ln r|+\varepsilon^{3}+\varepsilon^{4} r^{-1}+\varepsilon^{5} r^{-3}+\varepsilon^{6} r^{-4}\right), \quad r \rightarrow 0 \\
V_{1}(\xi ; s)=P_{1}^{(0)}(\xi ; s)+\sum_{j=1}^{5} \beta_{j}(s) \rho^{-j} \sin (j \varphi), \\
V_{2}(\xi ; s)=P_{2}^{(0)}(\xi ; s)+\sum_{j=1}^{3} \rho^{-2 j} P_{j+1}^{(j+1)}(\xi ; s), \\
V_{3}(\xi ; s)=P_{3}^{(0)}(\xi ; s)+\rho^{-2} P_{3}^{(2)}(\xi ; s)+P_{1,1}^{(2)}(\xi ; s) \ln \rho, \\
V_{3,1}(\xi ; s)=P_{1,1}^{(2)}(\xi ; s) .
\end{aligned}
$$

Следуя методу согласования асимптотических разложений, собственную функцию в малой окрестности кривой $\gamma$ будем искать в виде

$$
\begin{gathered}
\psi_{\varepsilon}(x) \approx \hat{v}_{\varepsilon}(\xi ; s)=\sum_{j=1}^{3} \varepsilon^{j} v_{j}(\xi ; s)+\varepsilon^{3} \ln \varepsilon v_{3,1}(\xi ; s), \\
v_{1}(\xi ; s)=V_{1}(\xi ; s)+O\left(\rho^{-6}\right), \\
v_{2}(\xi ; s)=V_{2}(\xi ; s)+\sum_{j=1}^{2} \delta^{j}(s) \rho^{-j} \sin (j \varphi)+O\left(\rho^{-3}\right), \\
v_{3}(\xi ; s)=V_{3}(\xi ; s)+O(1), \quad v_{3,1}(\xi ; s)=V_{3,1}(\xi ; s)+O\left(\rho^{-1}\right), \quad \rho \rightarrow \infty .
\end{gathered}
$$

Подставляя $(2.1),(3.16)$ в (1.2), переходя в полученном равенстве к переменным $\xi$ и приравнивая коэффициенты при одинаковых степенях $\varepsilon$ и $\ln \varepsilon$ (до $\varepsilon \ln \varepsilon$ включительно), получаем краевую задачу (3.3) для $v_{1}$ и следующие краевые задачи:

$$
\begin{gathered}
\Delta_{\xi} v_{2}=b_{0} \frac{\partial v_{1}}{\partial \xi_{1}}, \quad \xi_{2}>0, \quad v_{2}=0, \quad \xi \in \Gamma_{1}, \quad \frac{\partial v_{2}}{\partial \xi_{2}}=0, \quad \xi \in \gamma_{1}, \\
\Delta_{\xi} v_{3}=b_{1} \xi_{1} \frac{\partial v_{1}}{\partial \xi_{1}}+b_{0} \frac{\partial v_{2}}{\partial \xi_{1}}+h_{0} \frac{\partial^{2} v_{1}}{\partial s^{2}}-\lambda_{0} v_{1}, \quad \xi_{2}>0, \\
v_{3}=0, \quad \xi \in \Gamma_{1}, \quad \frac{\partial v_{3}}{\partial \xi_{2}}=0, \quad \xi \in \gamma_{1}, \\
\Delta_{\xi} v_{3,1}=0, \quad \xi_{2}>0, \quad v_{3,1}=0, \quad \xi \in \Gamma_{1}, \quad \frac{\partial v_{3,1}}{\partial \xi_{2}}=0, \quad \xi \in \gamma_{1} .
\end{gathered}
$$


Заметим, что функция $v_{1}$, определяемая равенством (3.4), удовлетворяет краевой задаче (3.3), а функция $v_{3,1}$, определяемая равенством

$$
v_{3,1}=a_{1}(s) \operatorname{Im} \sqrt{\left(\zeta-f_{1}\right)\left(\zeta-f_{2}\right)}
$$

где

$$
a_{1}(s)=\frac{1}{2}\left(\beta_{1} \frac{b_{0}^{2}}{4}-\beta_{1} \frac{b_{1}}{2}-\lambda_{0} \beta_{1}+h_{0} \beta_{1}^{\prime \prime}\right),
$$

удовлетворяет краевой задаче (3.20) и они имеют на бесконечности асимптотики (3.17).

Положим

$$
\begin{aligned}
\Pi= & C^{\infty}\left(\left\{\xi: \xi_{2} \geqslant 0, \xi \neq\left(f_{1}(s), 0\right), \xi \neq\left(f_{2}(s), 0\right)\right\}\right) \cap \\
& \cap W_{2}^{1}\left(\left\{\xi:\left|\xi-\frac{\left(f_{1}(s)+f_{2}(s)\right)}{2}\right|<f_{2}(s)-f_{1}(s), \xi_{2}>0\right\}\right) .
\end{aligned}
$$

Легко видеть, что $v_{1}$ и $v_{3,1}$ принадлежат П. Непосредственно проверяется, что функция

$$
v_{2}=\frac{b_{0}}{2} \xi_{1} v_{1}+\left(\alpha_{2}-\frac{\alpha_{1} b_{0}}{4}\right) \operatorname{Im}\left(\left(\zeta+\frac{f_{2}+f_{1}}{2}\right) \sqrt{\left(\zeta-f_{1}\right)\left(\zeta-f_{2}\right)}\right)
$$

является решением краевой задачи (3.18), принадлежит П и имеет на бесконечности асимптотику

$$
v_{2}(\xi ; s)=V_{2}(\xi ; s)+\sum_{j=1}^{2} \delta_{j}(s) \rho^{-j} \sin (j \varphi)+O\left(\rho^{-3}\right), \quad \rho \rightarrow \infty
$$

где $\delta_{j}(s)$ - некоторые явно вычисляемые функции.

Положив $\delta^{j}(s)=\delta_{j}(s)$ в (3.12), окончательно определим функции $\psi_{3}, \psi_{4}$.

Лемма 3.1. Существует решение краевой задачи (3.19) из П, имеющее на бесконечности асимптотику (3.17).

ДокАЗАТЕЛЬСтво. Предварительно рассмотрим краевую задачу

$$
\Delta_{\xi} v_{3}^{(1)}=v_{1}, \quad \xi_{2}>0, \quad v_{3}^{(1)}=0, \quad \xi \in \Gamma_{1}, \quad \frac{\partial v_{3}^{(1)}}{\partial \xi_{2}}=0, \quad \xi \in \gamma_{1} .
$$

Принимая во внимание (3.4) и (3.6), получаем, что функция

$$
\begin{aligned}
v_{3}^{(1)}= & \frac{\alpha_{1}}{4} \operatorname{Im}\left(\bar{\zeta} \int_{f_{2}}^{\zeta} \sqrt{\left(z-f_{1}\right)\left(z-f_{2}\right)} d z\right) \\
& +\frac{\beta_{1}}{4} \operatorname{Im}\left(\zeta \ln \left(\frac{2}{f_{2}-f_{1}}\left(\zeta-\frac{f_{1}+f_{2}}{2}+\sqrt{\left(\zeta-f_{1}\right)\left(\zeta-f_{2}\right)}\right)\right)\right)
\end{aligned}
$$

является решением краевой задачи (3.22), принадлежит П и имеет асимптотику

$$
v_{3}^{(1)}=\frac{\alpha_{1}}{8} \rho^{3} \sin \varphi+\frac{\beta_{1}}{2} \xi_{2} \ln \rho+\phi_{1}(s) \xi_{2}+O(1), \quad \rho \rightarrow \infty
$$


где $\phi_{1}(s) \in C^{\infty}(\gamma)$ - явно вычисляемая функция.

Далее получаем, что в силу (3.23) и (3.24) функция

$$
\begin{aligned}
v_{3}^{(2)}= & \frac{\partial^{2} v_{3}^{(1)}}{\partial s^{2}}+a_{1}^{(1)} \operatorname{Im} \sqrt{\frac{\zeta-f_{2}}{\zeta-f_{1}}}+a_{2}^{(1)} \operatorname{Im} \sqrt{\left(\frac{\zeta-f_{2}}{\zeta-f_{1}}\right)^{3}} \\
& +a_{1}^{(2)} \operatorname{Im} \sqrt{\frac{\zeta-f_{1}}{\zeta-f_{2}}}+a_{2}^{(2)} \operatorname{Im} \sqrt{\left(\frac{\zeta-f_{1}}{\zeta-f_{2}}\right)^{3}}
\end{aligned}
$$

где $a_{1}^{(1)}, a_{1}^{(2)}, a_{2}^{(1)}, a_{2}^{(2)}$ - некоторые явно вычисляемые функции, принадлежит П, а в силу (3.22) эта функция является решением краевой задачи

$$
\Delta_{\xi} v_{3}^{(2)}=\frac{\partial^{2} v_{1}}{\partial s^{2}}, \quad \xi_{2}>0, \quad v_{3}^{(2)}=0, \quad \xi \in \Gamma_{1}, \quad \frac{\partial v_{3}^{(2)}}{\partial \xi_{2}}=0, \quad \xi \in \gamma_{1} .
$$

Непосредственно проверяется, что функция

$$
v_{3}^{(3)}=\frac{b_{0}}{2} \xi_{1} v_{2}-\frac{b_{0}^{2}}{8} \xi_{1}^{2} v_{1}+\frac{b_{1}}{4} \xi_{1}^{2} v_{1}
$$

принадлежит П, является решением краевой задачи

$$
\begin{aligned}
& \Delta_{\xi} v_{3}^{(3)}=b_{1} \xi_{1} \frac{\partial v_{1}}{\partial \xi_{1}}+b_{0} \frac{\partial v_{2}}{\partial \xi_{1}}+\left(\frac{b_{1}}{2}-\frac{b_{0}^{2}}{4}\right) v_{1}, \quad \xi_{2}>0, \\
& v_{3}^{(3)}=0, \quad \xi \in \Gamma_{1}, \quad \frac{\partial v_{3}^{(3)}}{\partial \xi_{2}}=0, \quad \xi \in \gamma_{1},
\end{aligned}
$$

и имеет асимптотику

$$
\begin{aligned}
v_{3}^{(3)}= & \left(\frac{b_{0} \alpha_{2}}{4}+\frac{\alpha_{1}}{4}\left(\frac{b_{1}}{4}-\frac{b_{0}^{2}}{8}\right)\right) \rho^{3}(\sin \varphi+\sin 3 \varphi) \\
& +\frac{\beta_{1}}{16}\left(\frac{b_{0}^{2}}{2}+b_{1}\right) \rho(\sin \varphi+\sin 3 \varphi)+O(1), \quad \rho \rightarrow \infty .
\end{aligned}
$$

В силу краевых задач $(3.22),(3.25),(3.26)$ функция

$$
\begin{aligned}
v_{3}= & v_{3}^{(3)}-\left(-\frac{b_{0}^{2}}{4}+\frac{b_{1}}{2}+\lambda_{0}\right) v_{3}^{(1)}+h_{0} v_{3}^{(2)} \\
& +\operatorname{Im}\left(\left(A_{1}(s) \zeta^{2}+A_{1}(s) \frac{f_{2}+f_{1}}{2} \zeta+A_{2}(s)\right) \sqrt{\left(\zeta-f_{1}\right)\left(\zeta-f_{2}\right)}\right)
\end{aligned}
$$

где

$$
\begin{aligned}
A_{1}= & D_{1}^{(0)}-\frac{b_{0} \alpha_{2}}{4}-\frac{\alpha_{1}}{4}\left(\frac{b_{1}}{4}-\frac{b_{0}^{2}}{8}\right), \quad A_{2}=D_{1}^{(1)}+\left(-\frac{b_{0}^{2}}{4}+\frac{b_{1}}{2}+\lambda_{0}\right) \phi_{1} \\
& -h_{0} \phi_{1}^{\prime \prime}-\frac{\beta_{1}}{16}\left(\frac{b_{0}^{2}}{2}+b_{1}\right)+A_{1} \frac{3 f_{1}^{2}+3 f_{2}^{2}+2 f_{1} f_{2}}{8}
\end{aligned}
$$


является решением краевой задачи (3.19). Из (3.24), (3.27), (3.28) следует, что $v_{3}$ принадлежит П и имеет асимптотику (3.17). Лемма доказана.

Подставляя (3.17) в (3.16) и групируя коэффициенты при одинаковьх степенях $\varepsilon$ и $\ln \varepsilon$, получаем

$$
\begin{aligned}
\hat{v}_{\varepsilon}(\xi ; s)= & \varepsilon V_{1}(\xi ; s)+\varepsilon^{2}\left(V_{2}(\xi ; s)+\sum_{j=1}^{2} \delta_{j}(s) \rho^{-j} \sin (j \varphi)\right) \\
& +\varepsilon^{3} V_{3}(\xi ; s)+\varepsilon^{3} \ln \varepsilon V_{3,1}(\xi ; s)+O\left(\varepsilon \rho^{-6}+\varepsilon^{2} \rho^{-3}+\varepsilon^{3}+\rho^{-1} \varepsilon^{3} \ln \varepsilon\right) .
\end{aligned}
$$

Рассмотрим краевую задачу

$$
\begin{gathered}
(\Delta-1) \phi=-\left(1-\chi\left(r t \varepsilon^{-\alpha}\right)\right)\left(\frac{1}{H} \frac{\partial}{\partial s}\left(\frac{1}{H} \frac{\partial \hat{v}_{\varepsilon}}{\partial s}\right)-\frac{\partial^{2} v_{1}}{\partial s^{2}}\right), \quad x \in \Omega, \\
\phi=0, \quad x \in \Gamma_{\varepsilon}, \quad \frac{\partial \phi}{\partial \tau}=0, \quad x \in \gamma_{\varepsilon}
\end{gathered}
$$

где $2 / 3<\alpha<5 / 7, t$ - произвольная постоянная. Решение этой краевой задачи будем рассматривать в смысле теории распределений [13], т.е. под решением будем понимать функцию $\phi \in W_{2}^{1}(\Omega)$, равную нулю на $\Gamma_{\varepsilon}$, удовлетворяющую интегральному равенству

$$
-(\nabla \phi, \nabla \eta)-(\phi, \eta)=\left(\frac{1-\chi}{H} \frac{\partial \hat{v}_{\varepsilon}}{\partial s}, \frac{1}{H} \frac{\partial \eta}{\partial s}\right)-\left((1-\chi) \frac{\partial v_{1}}{\partial s}, \frac{\partial \eta}{\partial s}\right)
$$

при любом $\eta \in W_{2}^{1}(\Omega)$, равном нулю на $\Gamma_{\varepsilon}$. Здесь и всюду далее $(\cdot, \cdot)-$ скалярное произведение в $L_{2}(\Omega),\|\cdot\|_{0},\|\cdot\|_{1}-$ нормы в $L_{2}(\Omega)$ и $W_{2}^{1}(\Omega)$ соответственно. Из $(3.31)$ легко вьводится следующая оценка:

$$
\|\phi\|_{1} \leqslant C\left(\left\|(1-\chi) \frac{\partial \hat{v}_{\varepsilon}}{\partial s}\right\|_{0}+\left\|(1-\chi) \frac{\partial v_{1}}{\partial s}\right\|_{0}\right)
$$

где постоянная $C$ не зависит от $\varepsilon$ и $\eta$. Из данной оценки следует существование и единственность решения краевой задачи (3.30). Кроме того, на основе теорем о повьшении гладкости решений эллиптических краевых задач устанавливается принадлежность

$$
\phi \in W_{2}^{1}(\Omega) \cap C^{\infty}\left(\bar{\Omega} \backslash \partial \gamma_{\varepsilon}\right)
$$

Из (3.32), (3.29), (3.15) следует, в частности, что

$$
\|\phi\|_{0}=o\left(\varepsilon^{2}\right) \quad \text { при } \varepsilon \rightarrow 0 .
$$

Обозначим

$$
\widehat{\psi}_{\varepsilon}(x)=\chi\left(r t \varepsilon^{-\alpha}\right) \widehat{\psi}_{\varepsilon, 6}(x)+\left(1-\chi\left(r t \varepsilon^{-\alpha}\right)\right) \hat{v}_{\varepsilon}\left(y \varepsilon^{-1} ; s\right)+\phi(x, \varepsilon),
$$

где $2 / 3<\alpha<5 / 7, t>0$ - произвольная фиксированная постоянная. 
ТЕорема 3.1. Функиия $\widehat{\psi}_{\varepsilon}$ принадлежит $W_{2}^{1}(\Omega) \cap C^{\infty}\left(\bar{\Omega} \backslash \partial \gamma_{\varepsilon}\right)$ и является решением краевой задачи

$$
-\left(\Delta+\hat{\lambda}_{\varepsilon}\right) \widehat{\psi}_{\varepsilon}=\hat{f}_{\varepsilon}, \quad x \in \Omega, \quad \widehat{\psi}_{\varepsilon}=0, \quad x \in \Gamma_{\varepsilon}, \quad \frac{\partial \widehat{\psi}_{\varepsilon}}{\partial \tau}=0, \quad x \in \gamma_{\varepsilon}
$$

əде $\left\|\hat{f}_{\varepsilon}\right\|_{0}=o\left(\varepsilon^{2}\right),\left\|\widehat{\psi}_{\varepsilon}\right\|_{0}=1+o(1) n p u \varepsilon \rightarrow 0$.

ДокАЗАТЕльство. В силу $(2.1),(3.30)$ и лемм $2.3-3.2$ функция $\widehat{\psi}_{\varepsilon} \in W_{2}^{1}(\Omega) \cap C^{\infty}(\bar{\Omega} \backslash$ $\left.\partial \gamma_{\varepsilon}\right)$ и является решением краевой задачи (3.34) при

$$
\hat{f}_{\varepsilon}=-\sum_{j=1}^{4} f_{\varepsilon, j}(x)
$$

где

$$
\begin{aligned}
f_{\varepsilon, 1}(x)= & \Delta \chi\left(r t \varepsilon^{-\alpha}\right)\left(\widehat{\psi}_{\varepsilon, 6}-\hat{v}_{\varepsilon}\right)+2 \sum_{j=1}^{3} \chi\left(r t \varepsilon^{-\alpha}\right)_{x_{j}}\left(\widehat{\psi}_{\varepsilon, 6}-\hat{v}_{\varepsilon}\right)_{x_{j}}, \\
f_{\varepsilon, 2}(x)= & \chi\left(r t \varepsilon^{-\alpha}\right)\left(\lambda_{0} \sum_{n=3}^{6} \varepsilon^{n} \psi_{n}+\lambda_{2} \sum_{n=2}^{4} \varepsilon^{n+2} \psi_{n}\right), \\
f_{\varepsilon, 3}(x)= & \left(\hat{\lambda}_{\varepsilon}+1\right) \phi, \\
f_{\varepsilon, 4}(x)= & \left(1-\chi\left(r t \varepsilon^{-\alpha}\right)\right)\left(\sum_{j=1}^{3} \varepsilon^{2} \xi_{1}^{3-j} \delta_{j}^{(1)}\left(\xi_{1}, s, \varepsilon\right) \frac{\partial v_{j}}{\partial \xi_{1}} \varepsilon^{2} \ln \varepsilon \delta_{4}^{(1)}\left(\xi_{1}, s, \varepsilon\right) \frac{\partial v_{3,1}}{\partial \xi_{1}}\right. \\
& \left.+\lambda_{0} \sum_{j=2}^{3} \varepsilon^{j} v_{j}+\lambda_{2} \sum_{j=1}^{3} \varepsilon^{j+2} v_{j}+\varepsilon^{3} \ln \varepsilon \lambda_{0} v_{3,1}+\varepsilon^{5} \ln \varepsilon \lambda_{2} v_{3,1}\right),
\end{aligned}
$$

$\delta_{j}^{(k)}-$ ограниченные при $\rho<t \varepsilon^{\alpha-1}$ функции. В силу (3.17) функция $f_{\varepsilon, 4}=O\left(\varepsilon^{2} \rho^{2}\right)$ при $\rho \rightarrow \infty$. Так как supp $f_{\varepsilon, 4} \subset \Omega \cap S_{t^{-1} \varepsilon^{\alpha}}^{2}$, то $\left\|f_{\varepsilon, 4}\right\|_{0}^{2}=O\left(\varepsilon^{6 \alpha}\right)$. Из (3.14) и (3.29) следует, что при $t^{-1} \varepsilon^{\alpha}<r<2 t^{-1} \varepsilon^{\alpha}$ имеет место оценка $f_{\varepsilon, 1}=O\left(\varepsilon^{2 \alpha}+\varepsilon^{7-8 \alpha}\right)$. Так как $\operatorname{supp} f_{\varepsilon, 1} \subset S_{2 t^{-1} \varepsilon^{\alpha}}^{2} \backslash S_{\varepsilon^{\alpha}}^{2}$, то $\left\|f_{\varepsilon, 1}\right\|_{0}^{2}=O\left(\varepsilon^{-14 \alpha+14}+\varepsilon^{6 \alpha}\right)$. Из (2.8), (3.11), (3.13) следует, что $f_{\varepsilon, 2}=O\left(\varepsilon^{3} r^{-2}\right)$ при $r \rightarrow 0$. Так как supp $f_{\varepsilon, 2} \subset \Omega \backslash S_{2 t^{-1} \varepsilon^{\alpha}}^{2}$, то $\left\|f_{\varepsilon, 2}\right\|_{0}^{2}=$ $O\left(\varepsilon^{6-2 \alpha}\right)$. Из (3.33) следует, что $\left\|f_{\varepsilon, 3}\right\|_{0}=o\left(\varepsilon^{2}\right)$. Из оценок для $f_{\varepsilon, j}$ получаем, что $\left\|\hat{f}_{\varepsilon}\right\|_{0}^{2}=o\left(\varepsilon^{4}\right)$ при $2 / 3<\alpha<5 / 7$. Аналогично оценкам для $f_{\varepsilon, 2}, f_{\varepsilon, 3}$ получаем, что $\left\|\widehat{\psi}_{\varepsilon}\right\|_{0}=1+o(1)$ при $0<\alpha<1$. Теорема доказана.

\section{4. Обоснование асимптотик. В [12] было доказано следующее утверждение.}

ЛЕмма 4.1. Пусть $K$ - произвольный компакт в комплексной плоскости $\mathbb{C}$, не содержащий собственных значений (1.1), $f \in L_{2}(\Omega)$. Тогда существует $\varepsilon_{0}>0$ такое, что при любом $\varepsilon<\varepsilon_{0}$, любом $\lambda \in K$ существует единственное решение $u_{\varepsilon}$ краевой задачи

$$
-\Delta u_{\varepsilon}=\lambda u_{\varepsilon}+f \quad \text { в } \Omega, \quad \frac{\partial u_{\varepsilon}}{\partial \tau}=0 \quad \text { на } \gamma_{\varepsilon}, \quad u_{\varepsilon}=0 \quad \text { на } \Gamma_{\varepsilon}
$$

и справедлива равномерная по є и $\lambda$ оченка

$$
\left\|u_{\varepsilon}\right\|_{1} \leqslant C\|f\|_{0} .
$$

Для строгого обоснования асимптотик нам понадобится следующее утверждение. 
Лемма 4.2. Пусть $\lambda_{0}$ - простое собственное значение предельной задачи (1.1). Тогда

а) для нормированных в $L_{2}(\Omega)$ собственных функиий имеет место сходимость $\psi_{\varepsilon} \rightarrow \psi_{0}$ в $W_{2}^{1}(\Omega)$

б) при $\lambda$, близких $к \lambda_{0}$, для решений краевой задачи (4.1) справедлива равномерная по в и $\lambda$ оченка

$$
\left\|u_{\varepsilon}\right\|_{1} \leqslant \frac{C}{\left|\lambda_{\varepsilon}-\lambda\right|}\|f\|_{0} .
$$

ДокАЗАТЕЛЬСТво. Легко видеть, что в силу $(1.1),(1.2)$ для собственньх функций $\psi_{\varepsilon}, \psi_{0}$ справедливы равенства

$$
\left\|\nabla \psi_{\varepsilon}\right\|_{0}^{2}=\lambda_{\varepsilon}\left\|\psi_{\varepsilon}\right\|_{0}^{2}, \quad\left\|\nabla \psi_{0}\right\|_{0}^{2}=\lambda_{0}\left\|\psi_{0}\right\|_{0}^{2}
$$

Из леммы 1.1 следует, что сходятся правые части последних равенств, а значит,

$$
\left\|\psi_{\varepsilon}\right\|_{1} \rightarrow\left\|\psi_{0}\right\|_{1}
$$

Из (4.4) и слабой сходимости $\psi_{\varepsilon}$ в норме $W_{2}^{1}(\Omega)$ следует сильная сходимость в этой норме.

Обозначим через $S(t, z)$ открытьй круг на комплексной плоскости радиуса $t$ с центром в точке $z$. В силу лемм 1.1 и 4.1 существует достаточно малое $t$ такое, что при $\lambda \in \overline{S\left(t, \lambda_{0}\right)}$ справедливо представление

$$
u_{\varepsilon}=\frac{\psi_{\varepsilon}}{\lambda_{\varepsilon}-\lambda} \int_{\Omega} \psi_{\varepsilon} f d x+\tilde{u}_{\varepsilon}
$$

где $\tilde{u}_{\varepsilon}-$ голоморфная по $\lambda$ функция, ортогональная $\psi_{\varepsilon}$. Из (4.5) и (4.2) следует справедливость оценки

$$
\left\|\tilde{u}_{\varepsilon}\right\|_{1} \leqslant C_{1}\|f\|_{0}
$$

при $\lambda \in \partial S\left(t, \lambda_{0}\right)$. А так как $\tilde{u}_{\varepsilon}-$ голоморфная по $\lambda$ функция, то эта оценка верна и для $\lambda \in \overline{S\left(t, \lambda_{0}\right)}$. Из (4.6) и представления (4.5) следует (4.3).

Tеорема 4.1. Асимптотики собственного значения $\lambda_{\varepsilon}$ и соответствующей собственной функиии $\psi_{\varepsilon}$ имеют вид

$$
\begin{gathered}
\lambda_{\varepsilon}=\lambda_{0}+\varepsilon^{2} \lambda_{2}+o\left(\varepsilon^{2}\right), \\
\psi_{\varepsilon}(x)=c_{\varepsilon}^{1}\left(\psi_{0}(x)+\varepsilon^{2} \psi_{2}(x)+\varepsilon^{3} \psi_{3}(x)+\varepsilon^{4} \psi_{4}(x)+o\left(\varepsilon^{2}\right)\right) \quad s \Omega \backslash S_{\varepsilon^{\alpha}}^{2}, \\
\psi_{\varepsilon}(x)=c_{\varepsilon}^{2}\left(\varepsilon v_{1}(y ; s)+\varepsilon^{2} v_{2}(y ; s)+o\left(\varepsilon^{2}\right)\right) \quad в \Omega \cap S_{2 \varepsilon^{\alpha}}^{2},
\end{gathered}
$$

әде коэффиииенты определены равенствами (3.4), (3.10), (3.12), (3.21), а скалярные нормирующие множители $\left|c_{\varepsilon}^{i}\right| \rightarrow 1$ при $\varepsilon \rightarrow 0$. Равенства (4.7), (4.8) справедливь в норме $W_{2}^{1}$. 
ДокАЗАТЕльство. Из теоремы 3.1 следует, что $\widehat{\psi}_{\varepsilon}$ является решением краевой задачи (4.1) при $\lambda=\hat{\lambda}_{\varepsilon}, f=\hat{f}_{\varepsilon}$, причем

$$
\left\|\hat{f}_{\varepsilon}\right\|_{0, \Omega}=o\left(\varepsilon^{2}\right), \quad\left\|\widehat{\psi}_{\varepsilon}\right\|_{0, \Omega}=1+o(1)
$$

Из оценки (4.3) с учетом последних равенств заключаем, что $\left|\lambda_{\varepsilon}-\hat{\lambda}_{\varepsilon}\right|=o\left(\varepsilon^{2}\right)$.

Из (4.5) следует, что $\widehat{\psi}_{\varepsilon}$ может быть представлена в виде

$$
\widehat{\psi}_{\varepsilon}=\frac{\psi_{\varepsilon}}{\lambda_{\varepsilon}-\hat{\lambda}_{\varepsilon}} \int_{\Omega} \psi_{\varepsilon} \hat{f}_{\varepsilon} d x+\tilde{u}_{\varepsilon}=\hat{c}_{\varepsilon}(t) \psi_{\varepsilon}+\tilde{u}_{\varepsilon} .
$$

Из теоремы 3.1 и (4.6) следует, что

$$
\left\|\tilde{u}_{\varepsilon}\right\|_{1}=o\left(\varepsilon^{2}\right), \quad\left\|\widehat{\psi}_{\varepsilon}\right\|_{0}=1+o(1) .
$$

Поскольку $\left\|\psi_{\varepsilon}\right\|_{0}=1$, то отсюда и из (4.9) получаем, что $\left|\hat{c}_{\varepsilon}\right| \rightarrow 1$ при $\varepsilon \rightarrow 0$. Обозначим $\widetilde{\psi}_{\varepsilon}=\hat{c}_{\varepsilon} \psi_{\varepsilon}$. Так как $\widetilde{\psi}_{\varepsilon}-$ собственная функция, соответствующая $\lambda_{\varepsilon}$, и $\left\|\widehat{\psi}_{\varepsilon}-\widetilde{\psi}_{\varepsilon}\right\|_{1}=$ $\left\|\tilde{u}_{\varepsilon}\right\|_{1}=o\left(\varepsilon^{2}\right)$, то из сходимости $\left|\hat{c}_{\varepsilon}\right| \rightarrow 1$ следует, что $\widehat{\psi}_{\varepsilon}-$ асимптотика собственной функции с точностью до $o\left(\varepsilon^{2}\right)$. Положив $t=2$, получаем равенство (4.7) с $c_{\varepsilon}^{1}=\hat{c}_{\varepsilon}(2)$, a положив $t=1 / 2$ получаем (4.8) с $c_{\varepsilon}^{2}=\hat{c}_{\varepsilon}(1 / 2)$. Теорема 4.1 доказана.

Заметим, что область, где справедливо внешнее разложение (4.7), и область, где справедливо внутреннее разложение $(4.8)$, имеют пересечение $\Omega \cap S_{\varepsilon^{\alpha}}^{2} \backslash S_{2 \varepsilon^{\alpha}}^{2}$ и в этой области справедливо любое из разложений (4.7), (4.8).

5. Примеры и приложения построенных асимптотик. Пусть $\widetilde{\omega} \subset \mathbb{R}^{2}-$ односвязная ограниченная область с бесконечно дифференцируемой границей, $\lambda_{0}-$ простое собственное значение задачи Дирихле в $\Omega=\widetilde{\omega} \times(0, \pi)$. Так как в этом случае

$$
\psi_{0}(x)=\sqrt{\frac{2}{\pi}} \Phi\left(x^{\prime}\right) \sin \left(\varkappa x_{3}\right),
$$

где $x^{\prime}=\left(x_{1}, x_{2}\right), \varkappa>0$ - целое число, $\Phi$ - нормированная в $L_{2}(\widetilde{\omega})$ собственная функция задачи Дирихле для $-\Delta$ в $\widetilde{\omega}$, соответствуюшая простому собственному значению $\lambda_{0}-$ $\varkappa^{2}$, то, подставляя (5.1) в (3.10), получаем, что

$$
\lambda_{2}=-\frac{1}{4} \varkappa^{2} \int_{\gamma} \Phi^{2}\left(x^{\prime}(s)\right)\left(f_{2}(s)-f_{1}(s)\right)^{2} d s .
$$

Если $f_{1}(s)=-1, f_{2}(s)=1$ (т.е. $\gamma_{\varepsilon}$ - симметричная полоса), то из (5.2) следует

$$
\lambda_{2}=-\varkappa^{2} \int_{\gamma} \Phi^{2}\left(x^{\prime}(s)\right) d s .
$$

Если $\widetilde{\omega}$ - круг радиуса $R_{1}$ с центром в начале координат, а $\gamma$ - окружность радиуса $r_{1}$, то

$$
\Phi\left(x^{\prime}\right)=\frac{I_{0}\left(\nu_{j}^{(0)}\left|x^{\prime}\right| / R_{1}\right)}{\sqrt{\pi} I_{0}^{\prime}\left(\nu_{j}^{(0)}\right)}
$$


где $\nu_{j}^{(0)}-$ корни функции Бесселя нулевого порядка $I_{0}(\nu)$. Подставляя это равенство в $(5.2)$ и учитьвая, что $y_{1}=\left|x^{\prime}\right|-r_{1}$, получаем

$$
\lambda_{2}=-\frac{1}{4 \pi^{2} R_{1}^{2}}\left(\frac{\varkappa}{I_{0}^{\prime}\left(\nu_{j}^{(0)}\right)} I_{0}\left(\nu_{j}^{(0)} \frac{r_{1}}{R_{1}}\right)\right)^{2} \int_{\gamma}\left(f_{2}(s)-f_{1}(s)\right)^{2} d s .
$$

И, наконец, если к тому же $f_{1}(s)=-1, f_{2}(s)=1$, то из последней формулы следует

$$
\lambda_{2}=-\frac{2 r_{1}}{\pi R_{1}^{2}}\left(\frac{\varkappa}{I_{0}^{\prime}\left(\nu_{j}^{(0)}\right)} I_{0}\left(\nu_{j}^{(0)} \frac{r_{1}}{R_{1}}\right)\right)^{2}
$$

Построенные асимптотики позволяют получить оценки для собственных значений лапласиана при других типах сингулярных возмущений.

Будем считать, что собственные значения краевых задач (1.2) и (1.1) занумерованы в порядке неубьвания, причем каждое повторяется столько раз, какова его кратность:

$$
\lambda_{\varepsilon}^{1} \leqslant \lambda_{\varepsilon}^{2} \leqslant \cdots \leqslant \lambda_{\varepsilon}^{k} \leqslant \cdots, \quad \lambda_{0}^{1} \leqslant \lambda_{0}^{2} \leqslant \cdots \leqslant \lambda_{0}^{k} \leqslant \cdots .
$$

Соответствующие собственные функции будем предполагать ортонормированными в $L_{2}(\Omega)$.

Пусть множества $\gamma_{\varepsilon}^{1}, \gamma_{\varepsilon}^{2} \subset \Gamma$, причем

$$
\gamma_{\varepsilon}^{i}=\left\{\left(y_{1}, 0, s\right): \varepsilon f_{1}^{i}(s)<y_{1}<\varepsilon f_{2}^{i}(s)\right\}, \quad i=1,2,
$$

$f_{1}^{i}(s), f_{2}^{i}(s) \in C^{\infty}(\gamma), i=1,2$. Обозначим через $\gamma_{\varepsilon}$ произвольную область такую, что $\gamma_{\varepsilon}^{1} \subset \gamma_{\varepsilon} \subset \gamma_{\varepsilon}^{2}$. Тогда для $\lambda_{\varepsilon}^{k}$ верны неравенства

$$
\lambda_{\varepsilon, 2}^{k} \leqslant \lambda_{\varepsilon}^{k} \leqslant \lambda_{\varepsilon, 1}^{k}
$$

где $\lambda_{\varepsilon, i}^{k}, \quad i=1,2,-$ собственные значения краевой задачи $(1.2)$ с $\gamma_{\varepsilon}=\gamma_{\varepsilon}^{i}$, расположенные в порядке неубывания с учетом кратности. Так как $\lambda_{\varepsilon, i}^{k} \rightarrow \lambda_{0}^{k}$, то в силу (5.3) и теоремы 4.1 вьполнены оценки

$$
M^{2} \varepsilon^{2}+o\left(\varepsilon^{2}\right) \leqslant \lambda_{0}-\lambda_{\varepsilon}^{k} \leqslant M^{1} \varepsilon^{2}+o\left(\varepsilon^{2}\right)
$$

где

$$
M^{i}=-\frac{\pi}{8} \int_{\gamma}\left(\frac{\partial \psi_{0}(x(s))}{\partial \tau}\right)^{2}\left(f_{2}^{i}(s)-f_{1}^{i}(s)\right)^{2} d s .
$$

Рассмотрим следующую сингулярно возмущенную краевую задачу:

$$
-\Delta \widetilde{\psi}_{\varepsilon}=\widetilde{\lambda}_{\varepsilon} \widetilde{\psi}_{\varepsilon} \quad \text { в } \Omega, \quad \widetilde{\psi}_{\varepsilon}=0 \text { на } \Gamma_{\varepsilon}, \quad \frac{\partial \widetilde{\psi}_{\varepsilon}}{\partial \tau}+\sigma(x) \widetilde{\psi}_{\varepsilon}=0 \text { на } \gamma_{\varepsilon}
$$

где $\sigma(x) \in C^{\infty}(\omega), \sigma(x) \geqslant 0$. Тогда из вариационных свойств собственных значений и собственных функций следует, что

$$
\lambda_{\varepsilon}^{k} \leqslant \widetilde{\lambda}_{\varepsilon}^{k} \leqslant \lambda_{0}^{k}
$$


где $\widetilde{\lambda}_{\varepsilon}^{k}$ - собственные значения краевой задачи (5.4), расположенные в порядке неубывания с учетом кратности. Тогда из теоремы 4.1 следует

$$
0 \leqslant \lambda_{0}^{k}-\widetilde{\lambda}_{\varepsilon}^{k} \leqslant C \varepsilon^{2} .
$$

Заметим, что в [14] рассматривалась задача в цилиндре со сменой типа граничного условия на периодически чередуюшихся узких полосках, расположенных на боковой поверхности. "Предельным" (усредненньм) граничньп условием было также условие Дирихле. Существенным отличием настоящей работы от [14] является тот факт, что в [14] асимптотики строились на основе метода составных разложений [15]. В задаче же, рассмотренной в настоящей работе, техника пограничного слоя не дает требуемого ответа. Поэтому необходимьм оказалось применение метода согласования асимптотических разложений.

В заключение хотелось бы выразить признательность Р. Р. Гадыльшину и Д. И. Борисову за обсуждение результатов и полезные замечания.

\section{СПИСОК ЦИТИРОВАННОЙ ЛИТЕРАТУРЫ}

[1] Ильин А. М. Согласование асимптотических разложений решений краевых задач. М.: Наука, 1989.

[2] Самарский А. А. О влиянии закрепления на собственные частоты замкнутых объемов // Докл. АН СССР. 1948. Т. 63. С. 631-634.

[3] Днестровский Ю. Н. Об изменении собственных чисел при изменении границы областей // Вестн. МГУ. 1964. №9. С. 61-74.

[4] Ozawa S. Singular variation of domains and eigenvalues of the Laplacian // Duke Math. J. 1981. V. 48. P. 767-778.

[5] Мазья В. Г., Назаров С. П., Пламеневский Б. А. Асимптотические разложения собственных чисел краевых задач для оператора Лапласа в областях с мальми отверстиями // Изв. АН СССР. Сер. матем. 1984. Т. 48. № 2. С. 347-371.

[6] Гадыльшин Р. Р., Ильин А. М. Асимптотика собственных значений задачи Дирихле в области с узкой щелью // Матем. сб. 1998. Т. 189. № 4. С. 25-48.

[7] Ильин А. М., Гадыльшин Р. Р. О границе применимости регулярной теории возмущений для мембраны с узкой щелью // Докл. РАН. 2001. Т. 380. № 2. С. 172-175.

[8] Гадыльшин P. P. Асимптотика собственного значения сингулярно возмущенной эллиптической задачи с малым параметром в граничном условии // Дифференц. уравнения. 1986. T. 22. № 4. C. $640-652$.

[9] Гадыльшин Р. Р. Расщепление кратного собственного значения задачи Дирихле для оператора Лапласа при сингулярном возмущении граничного условия // Матем. заметки. 1992. T. 52. № 4. C. $42-55$.

[10] Гадыльшин Р. Р. О возмущении спектра лапласиана при смене типа граничного условия на малой части границы // ЖВМиМФ. 1996. № 7. С. 77-88.

[11] Аргатов И. И., Назаров С. А. Асимптотическое решение задачи Синьорини с препятствием на тонком продолговатом множестве // Матем. сб. 1996. Т. 187. № 10. С. 3-32.

[12] Планида М. Ю. О сходимости решений сингулярно возмущенных краевых задач для лапласиана // Матем. заметки. 2002. Т. 71. №6. С. 867-877.

[13] Ладыженская О. А., Уральцева Н. Н. Линейные и квазилинейные уравнения эллиптического типа. М.: Наука, 1977.

[14] Борисов Д. И. О сингулярно возмущенной краевой задаче для лапласиана в цилиндре // Дифференц. уравнения. 2002. Т. 38. № 8. С. 1071-1078.

[15] Вишик М. И., Люстерник Л. А. Регулярное вырождение и пограничньй слой для линейных дифференциальных уравнений с малым параметром // УМН. 1957. Т. 12. №5. C. $3-122$.

Башкирский государственный педагогический университет, Уфа 